\title{
GRB 010222: A BURST WITHIN A STARBURST
}

\author{
D. A. Frail ${ }^{1}$ F. Bertoldi, ${ }^{2}$ G. H. Moriarty-Schieven, ${ }^{3}$ E. Berger,${ }^{4}$ P. A. Price ${ }^{4}$ J. S. Bloom,${ }^{4}$ R. Sari, ${ }^{5}$ \\ S. R. Kulkarni, ${ }^{4}$ C. L. Gerardy,${ }^{6}$ D. E. Reichart, ${ }^{4}$ S. G. Djorgovisi,${ }^{4}$ T. J. Galama, ${ }^{4}$ F. A. Harrison, ${ }^{4}$ F. Walter, ${ }^{4}$ \\ D. S. Shepherd, ${ }^{1}$ J. Halpern, ${ }^{7}$ A. B. PeCK, ${ }^{2}$ K. M. Menten, ${ }^{2}$ S. A. Yost,${ }^{4}$ and D. W. Fox ${ }^{4}$ \\ Received 2001 July 24 ; accepted 2001 October 3
}

\begin{abstract}
We present millimeter- and submillimeter-wavelength observations and near-infrared $K$-band imaging toward the bright gamma-ray burst GRB 010222. Over seven different epochs, a constant source was detected with an average flux density of $3.74 \pm 0.53 \mathrm{mJy}$ at $350 \mathrm{GHz}$ and $1.05 \pm 0.22 \mathrm{mJy}$ at $250 \mathrm{GHz}$, giving a spectral index $\alpha=3.78 \pm 0.25$ (where $F \propto v^{\alpha}$ ). We rule out the possibility that this emission originated from the burst or its afterglow, and we conclude that it is due to a dusty, high-redshift starburst galaxy (SMM J14522+4301). We argue that the host galaxy of GRB 010222 is the most plausible counterpart of SMM J14522 + 4301, based in part on the centimeter detection of the host at the expected level. The optical/near-IR properties of the host galaxy of GRB 010222 suggest that it is a blue sub- $L_{*}$ galaxy, similar to other GRB host galaxies. This contrasts with the enormous far-infrared luminosity of this galaxy based on our submillimeter detection $\left(L_{\mathrm{Bol}} \approx 4 \times 10^{12} L_{\odot}\right)$. We suggest that this GRB host galaxy has a very high star formation rate, SFR $\approx 600 M_{\odot} \mathrm{yr}^{-1}$, most of which is unseen at optical wavelengths.
\end{abstract}

Subject headings: galaxies: starburst — gamma rays: bursts

\section{INTRODUCTION}

GRB 010222 was detected on 2001 February 22.31 UT by the gamma-ray burst (GRB) monitor on the BeppoSAX satellite and localized to an error circle of 2.5 by the Wide Field Camera (Piro 2001a, 2001b). A bright optical afterglow candidate was quickly identified by Henden (2001) and subsequently confirmed by follow-up optical and radio observations (McDowell 2001; Berger \& Frail 2001). Absorption lines measured by Jha et al. (2001) yielded a lower redshift limit to the GRB of $z=1.477$, which they argued to be the redshift of the GRB host galaxy based on the unusually strong equivalent widths of the metallic lines. Since the burst and its afterglow were bright, we initiated GRB target-of-opportunity programs at several different telescope facilities (Frail et al. 2000b).

In this paper we report on observations of GRB 010222 , which, contrary to expectations, was detected as a constant millimeter and submillimeter source while the flux density at radio and optical wavelengths showed the usual declining behavior expected from the afterglow. After considering several different origins for the emission, we conclude that we have detected the host galaxy of the burst, which radiates primarily in the infrared (IR) and submillimeter because of the dust and gas heated by intense star formation.

\footnotetext{
${ }^{1}$ National Radio Astronomy Observatory, P.O. Box O, Socorro, NM 87801.

${ }^{2}$ Max-Planck-Institut für Radioastronomie, Auf dem Huegel 69, D-53121 Bonn, Germany.

${ }^{3}$ National Research Council of Canada, Joint Astronomy Centre, 660 North A'ohoku Place, Hilo, HI 96720.

${ }^{4}$ California Institute of Technology, Palomar Observatory, MS 105-24, Pasadena, CA 91125.

${ }^{5}$ California Institute of Technology, Theoretical Astrophysics, MS 130-33, Pasadena, CA 91125.

${ }^{6}$ Department of Physics and Astronomy, 6127 Wilder Laboratory, Dartmouth College, Hanover, NH 03755-3528.

${ }^{7}$ Astronomy Department, Columbia University, 550 West 120th Street, New York, NY 10027.
}

\section{RADIO OBSERVATIONS}

James Clerk Maxwell Telescope $(J C M T)^{8}$.- A frequencyordered $\log$ of all observations and flux density measurements is given in Table 1. Observations were made in the 350 and $660 \mathrm{GHz}$ bands using the Submillimeter CommonUser Bolometer Array (SCUBA; Holland et al. 1999). In this mode, the secondary mirror is chopped between the on-source position and a position $60^{\prime \prime}$ away in azimuth in order to remove sky emission. The angular resolution of the telescope is $\sim 14^{\prime \prime}$ at $350 \mathrm{GHz}$ and $\sim 8^{\prime \prime}$ at $660 \mathrm{GHz}$. The pointing was checked approximately once per hour on a nearby blazar (JVAS $1419+5423$ ) that is located less than $13^{\circ}$ from the GRB. The pointing was generally found to vary by less than $2^{\prime \prime}-3^{\prime \prime}$. On all days where observations were taken, the focus was checked at least every 2-4 hours, usually on the (local) pointing source.

The data were reduced using the SCUBA User Reduction Facility (Jenness \& Lightfoot 1998). The raw signals were flat-fielded to account for the small differences in bolometer response, extinction corrected, and despiked to remove anomalous signals above the $3 \sigma$ level. Short-timescale sky variations were also removed using pixels around the edge of the array containing no source emission (Jenness, Lightfoot, \& Holland 1998). A flux calibration factor was then applied to convert to Jy. Flux calibration factors of $197 \pm 13$ and $384 \pm 82 \mathrm{Jy} \mathrm{V}^{-1}$ were applied to the 350 and $660 \overline{\mathrm{GHz}}$ data, respectively (Coulson 2000). ${ }^{9}$

The atmospheric opacity at $220 \mathrm{GHz}$ was measured every 10 minutes using a tipping radiometer mounted near and operated by the Caltech Submillimeter Observatory. These values were then extrapolated to 350 and $660 \mathrm{GHz}$ and used

\footnotetext{
${ }^{8}$ The JCMT is operated by the Joint Astronomy Centre on behalf of the Particle Physics and Astronomy Research Council of the UK, the Netherlands Organization for Scientific Research, and the National Research Council of Canada.

${ }^{9}$ JAC Documentation, SCUBA Flux Conversion Factors (Coulson 2000), is available at http://www.jach.hawaii.edu/JACpublic/JCMT/ Continuum_observing/SCUBA/astronomy/calibration/gains.html.
} 
TABLE 1

SubMILlimeter AND Millimeter ObSERVATIONS OF GRB 010222

\begin{tabular}{|c|c|c|c|c|c|}
\hline $\begin{array}{l}\text { Frequency } \\
\qquad(\mathrm{GHz})\end{array}$ & Start Date & UT Start-End & $\begin{array}{l}\text { On-Source Time } \\
\text { (s) }\end{array}$ & $\begin{array}{l}\text { Flux } \pm \text { rms } \\
\quad(\mathrm{mJy})\end{array}$ & $\tau$ \\
\hline \multicolumn{6}{|c|}{ JCMT } \\
\hline 660. & Feb 24 & $16: 17-17: 56$ & 3600 & $<37.8(3 \sigma)$ & 0.055 \\
\hline $350 \ldots \ldots \ldots$ & Feb 22 & $13: 03-18: 39$ & 10800 & $4.34 \pm 1.09$ & 0.11 \\
\hline $350 \ldots \ldots \ldots$ & Feb 23 & $11: 08-19: 30$ & 18900 & $3.36 \pm 0.72$ & 0.09 \\
\hline $350 \ldots \ldots \ldots$ & Feb 24 & $16: 17-19: 29$ & 6300 & $4.04 \pm 1.14$ & 0.055 \\
\hline $350 \ldots \ldots \ldots$ & Mar 1 & $11: 54-12: 50$ & 2070 & $1.14 \pm 2.06$ & 0.08 \\
\hline $350 \ldots \ldots \ldots$ & Mar 2 & $14: 54-18: 05$ & 7200 & $0.63 \pm 0.94$ & 0.05 \\
\hline $350 \ldots \ldots \ldots$ & Mar 12 & $16: 18-19: 07$ & 5400 & $3.98 \pm 1.25$ & 0.05 \\
\hline \multicolumn{6}{|c|}{ IRAM } \\
\hline $250 .$. & Feb 22 & $22: 33-06: 43$ & 8850 & $1.32 \pm 0.54$ & $0.45-0.51$ \\
\hline $250 \ldots \ldots \ldots$ & Feb 25 & $00: 28-07: 55$ & 9140 & $1.09 \pm 0.32$ & $0.17-0.29$ \\
\hline $250 \ldots \ldots \ldots$ & Feb 26 & $01: 12-08: 52$ & 8160 & $1.00 \pm 0.33$ & $0.23-0.28$ \\
\hline $250 \ldots \ldots \ldots$ & Mar 13 & $07: 55-08: 09$ & 660 & $0.91 \pm 0.94$ & 0.08 \\
\hline $250 \ldots \ldots \ldots$ & Mar 14 & $05: 31-06: 00$ & 1330 & $1.31 \pm 0.61$ & 0.12 \\
\hline $250 \ldots \ldots \ldots$ & Mar 18 & $22: 33-22: 48$ & 830 & $0.78 \pm 1.10$ & 0.17 \\
\hline $250 \ldots \ldots \ldots$ & May 5 & $22: 57-03: 18$ & 2300 & $1.13 \pm 0.48$ & $0.14-0.16$ \\
\hline \multicolumn{6}{|c|}{ OVRO } \\
\hline $221 \ldots \ldots \ldots$ & Feb 23 & $07: 26-17: 40$ & 18000 & $1.15 \pm 5.00$ & 0.25 \\
\hline $98.5 \ldots \ldots$. & Feb 22 & $14: 31-19: 44$ & 8400 & $0.30 \pm 2.00$ & $>0.25$ \\
\hline $98.5 \ldots \ldots$. & Feb 23 & $07: 26-17: 40$ & 18000 & $-0.30 \pm 0.80$ & 0.25 \\
\hline
\end{tabular}

NoTE.-From left to right the columns are the sky frequency at which the observations were made, the starting date (2001), the UT start and end time, the time on source in seconds, the peak flux density at the position of GRB 010222 and the rms noise in mJy, and the zenith atmospheric opacity at $220 \mathrm{GHz}(250$ GHz at IRAM). See $\S 2$ for more details on the data calibration.

to correct for atmospheric extinction (Archibald, Wagg, \& Jenness 2000). ${ }^{10}$

IRAM $30 \mathrm{~m}$ telescope (IRAM).-Observations were made using the Max-Planck Millimeter Bolometer (MAMBO; Kreysa et al. 1998) array at the IRAM $30 \mathrm{~m}$ telescope on Pico Veleta, Spain. MAMBO is a 37 element bolometer array sensitive between 190 and $315 \mathrm{GHz}$. The larger than half-peak sensitivity range is $210-290 \mathrm{GHz}$, with an effective center frequency of $\sim 250 \mathrm{GHz}$ for steepspectrum sources. The beam for the feed horn of each bolometer is matched to the telescope beam of 10".6 and, the bolometers are arranged in a hexagonal pattern with a beam separation of $22^{\prime \prime}$. Observations were made in standard on-off mode with $2 \mathrm{~Hz}$ chopping of the secondary by $32^{\prime \prime}$.

The telescope pointing was checked every $\sim 20$ minutes with JVAS $1506+4239$, a point source $\sim 2.5$ from the optical transient (OT) position. The flux of the pointing source was monitored and appeared stable at $0.15 \mathrm{Jy}$. The target pointing accuracy is better than $2^{\prime \prime}$. GRB 010222 was positioned on the central bolometer of the array and the telescope was nodded after each $10 \mathrm{~s}$ of integration so that the previous "off" beam becomes the "on" beam. Each scan of $1610 \mathrm{~s}$ subscans lasts 4 minutes, of which $80 \mathrm{~s}$ of integration falls on the source, $80 \mathrm{~s}$ off source, and about 80 $\mathrm{s}$ are lost to move the telescope and start integration. Gain calibration was performed using observations of planets, resulting in a flux calibration factor of 12,500 counts per jansky, which is estimated to be accurate to $15 \%$. Opacity

\footnotetext{
10 JAC Documentation, Calculating Sky Opacities: A Re-analysis for SCUBA Data (Archibald, Wagg, \& Jenness 2000), is available at http:// www.jach.hawaii.edu/JACdocs/JCMT/SCD/SN/002.2/tau_analysis.html.
}

corrections were made from frequent skydips. A gainelevation correction was applied.

The data were reduced using the MOPSI software package (Zylka 1998). The average flux densities found at the target position along with other observational parameters are given in Table 1.

Owens Valley Radio Observatory Interferometer $(O V R O)$.- Observations were made on two nights at a central frequency of $98.48 \mathrm{GHz}$ with a $1 \mathrm{GHz}$ wide band. On the second night, weather conditions improved sufficiently to add a second frequency at $220.73 \mathrm{GHz}$ with a 2 $\mathrm{GHz}$ wide band. Phase calibration was performed using the source $3 \mathrm{C} 345$, while observations of Neptune provided the flux density calibration scale with an estimated uncertainty of $\sim 15 \%$. See Shepherd et al. (1998) for further details on calibration and imaging. No source was detected on either night.

\section{OPTICAL AND NEAR-IR OBSERVATIONS}

MDM Observatory.-The position of GRB 010222 was observed in the $K$ band using the TIFKAM infrared imager/spectrograph on the $2.4 \mathrm{~m}$ Hiltner telescope at MDM Observatory on 2001 April 11. This instrument uses a $512 \times 1024 \mathrm{InSb}$ detector and covers a $2.5 \times 5^{\prime}$ field of view. A total of 135 dithered $30 \mathrm{~s}$ exposures were skysubtracted and combined to yield a total exposure of 67.5 minutes. The seeing was $0.8-0.9$, and the air mass ranged from 1.02 to 1.14 . Nine standard stars from the list of Persson et al. (1998) were observed throughout the night. From these observations we find that the photometric zeropoint accuracy is $0.1 \mathrm{mag}$, probably due to the presence of light cirrus. 
TABLE 2

Magnitudes and Colors of $K$-BAnd Selected GalaXies WITHIN THE SCUBA BEAM

\begin{tabular}{ccccc}
\hline \hline Galaxy & $\begin{array}{c}\text { Aperture } \\
(\operatorname{arcsec})\end{array}$ & F814W & $K^{\mathrm{a}}$ & F814W $-K$ \\
\hline A $\ldots \ldots \ldots$. & 1.42 & $21.786 \pm 0.010$ & $18.233 \pm 0.034$ & $3.553 \pm 0.035$ \\
B $\ldots \ldots \ldots$. & 1.60 & $22.931 \pm 0.028$ & $18.398 \pm 0.035$ & $4.533 \pm 0.044$ \\
C $\ldots \ldots \ldots$. & 1.96 & $22.124 \pm 0.018$ & $19.049 \pm 0.041$ & $3.074 \pm 0.044$ \\
D ........ & 1.42 & $24.934 \pm 0.151$ & $21.102 \pm 0.113$ & $3.832 \pm 0.188$ \\
Host...... & 0.45 & $25.58 \pm 0.13$ & $22.745 \pm 0.274^{\mathrm{b}}$ & $2.834 \pm 0.303$ \\
\hline
\end{tabular}

${ }^{a}$ The error estimates do not include a zero-point uncertainty of $0.1 \mathrm{mag}$ (see $\S 3$ ).

b The host galaxy of GRB 010222 in the $K$ band is contaminated to some extent by the afterglow. An estimate from broadband modeling is that the afterglow contributes approximately $50 \%$ of the total flux at this epoch. There is no afterglow component in the F814W band, since the host and afterglow were fit independently. After correcting for the afterglow contamination, we find that the host color is bluer, i.e., $\mathrm{F} 814 \mathrm{~W}-K \simeq 2.1$.

The magnitudes of several objects in the neighborhood of the GRB host galaxy were measured. For reference, a magnitude $K=16.507 \pm 0.033$ was determined for star "E" (Fig. 3), which lies 10" south and 5".3 east of GRB 010222. Based on this calibration and the photometry of the faintest visible objects near the OT position, a $4 \sigma$ upper limit $K>19.6$ was derived at the position of GRB 010222 .

Keck Observatory.-The location of GRB 010222 was observed with the NIRSPEC slit-viewing camera on Keck II $^{11}$ on 2001 April 27 UT, approximately 64 days after the GRB. The observations consisted of a total of 58 minutes integration over 20 individual dithered frames with the air mass ranging from 1.3 to 1.7 . The conditions were photometric with $\sim 0$ " 45 seeing. The images were sky-subtracted and combined using IRAF/DIMSUM. ${ }^{12}$

The MDM-calibrated magnitude of star E (see above) was used as the basis of the magnitude zero point. The host of GRB 010222 is detected at the $\sim 3.6 \sigma$ level (see Fig. 3). For completeness the four other objects in the $K$-band image that lie within a 7" radius are indicated in Figure 3. Table 2 contains the results of the $K$-band and F814W photometry and the F814W $-K$ colors (see below). Aperture photometry was performed on each of these sources with circular apertures whose radii were chosen to include most of the object flux, based on a curve of growth.

In order to derive colors for these galaxies, matching apertures were used (with recentering allowed) to photometer Hubble Space Telescope (HST) + Wide Field Planetary Camera 2 (WFPC2) F814W images of the field. These HST data were taken over four epochs and are part of a multiepoch broadband monitoring of the afterglow of GRB 010222. Full details of the HST observations can be found in T. J. Galama et al. (2001, in preparation).

\section{THE ORIGIN OF THE SUBMILLIMETER AND MILLIMETER EMISSION}

Emission was detected toward GRB 010222 at 250 and $350 \mathrm{GHz}$ (see Table 1). In contrast to the steady decay of the

\footnotetext{
${ }^{11}$ The W. M. Keck Observatory is operated by the California Association for Research in Astronomy, a scientific partnership among California Institute of Technology, the University of California, and the National Aeronautics and Space Administration.

${ }^{12}$ See http://iraf.noao.edu/. The DIMSUM package was developed by P. Eisenhardt, M. Dickinson, S. A. Standford, and J. Ward with assistance from $F$. Valdes.
}

centimeter and optical emission, the submillimeter flux at $350 \mathrm{GHz}$ remains remarkably constant from $6 \mathrm{hr}$ after the burst until 18 days later (Fig. 1). The noticeable exception to this behavior occurs on day 8 , when the measured flux density lies $2.4 \sigma$ below the average of all measurements. The data were examined carefully for errors and inconsistencies in the calibration and data taking, but apart from some problems with the telescope focus at this time, we can find no instrumental origin for the deviation. Nevertheless, significant fluctuations in photometric measurements taken with single-dish submillimeter telescopes are not uncommon (R. Ivison 2001, private communication). For example, the $350 \mathrm{GHz}$ flux density of the $z=1.44$ ultraluminous starburst galaxy ERO J164502+4626.4, made in photometry mode and reported by Cimatti et al. (1998), is twice the value obtained in mapping mode (Dey et al. 1999). A search for variability was carried out within individual days, but no statistically significant evidence for any variations was

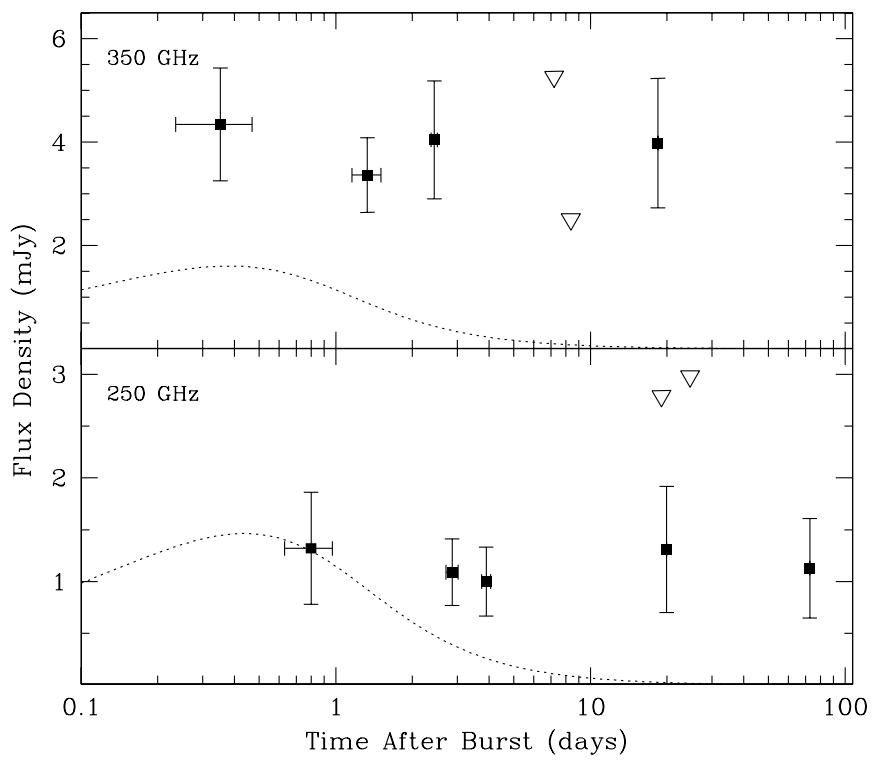

FIG. 1.-Flux density measurements toward GRB 010222 taken with the SCUBA bolometer array on the James Clerk Maxwell Telescope (top panel) and with MAMBO on the IRAM $30 \mathrm{~m}$ telescope (bottom panel). Upper limits are shown as open triangles and are plotted as the peak flux density at the location of the afterglow plus 2 times the rms noise. The observing frequency is shown in the upper left corner of each panel. The dotted lines are afterglow light curves derived from a global fit to the entire broadband data set (see T. J. Galama et al. 2001, in preparation). 
found. Adding the $350 \mathrm{GHz}$ data from all days together gives an average flux of $3.13 \pm 0.47 \mathrm{mJy}$. Excluding the low measurement gives an average of $3.74 \pm 0.53 \mathrm{mJy}$.

Independent support that the source is constant comes from the IRAM $250 \mathrm{GHz}$ measurements. Although the flux level is weaker than at $350 \mathrm{GHz}$ (Fig. 1), the average flux density at $250 \mathrm{GHz}$ over the same time interval (found by averaging all observations while weighting each scan with the inverse square of its average noise level) was $1.05 \pm 0.22$ mJy. A later measurement in 2001 May 5 gives a consistent value of $1.13 \pm 0.48 \mathrm{mJy}$. The 350 to $250 \mathrm{GHz}$ flux ratio of these average flux density values implies a steep spectral index $\alpha=3.78 \pm 0.25$ (where $F \propto \nu^{\alpha}$ ).

The constancy and steep spectral slope of the submillimeter and millimeter emission toward GRB 010222 are anomalous compared to the behavior of past GRBs and when compared to observations of this event at other wavelengths. In particular, the temporal behavior is difficult to reconcile with the expectations of the simplest afterglow models. This point is illustrated in Figure 1, where we show the deviation of the 250 and $350 \mathrm{GHz}$ measurements from the predicted afterglow light curves, as determined by broadband fitting of the radio, optical, and X-ray data (T. J. Galama et al. 2001, in preparation).

Similar difficulties are posed by the peculiar spectral slope (see Fig. 2), whose value between 250 and $350 \mathrm{GHz}$ is $\alpha=3.78 \pm 0.25$. Free-free or synchrotron self-absorption (Waxman 1997; Katz \& Piran 1997) in the millimeter band can lead to steep slopes with $\alpha=2-2.5$. This explanation

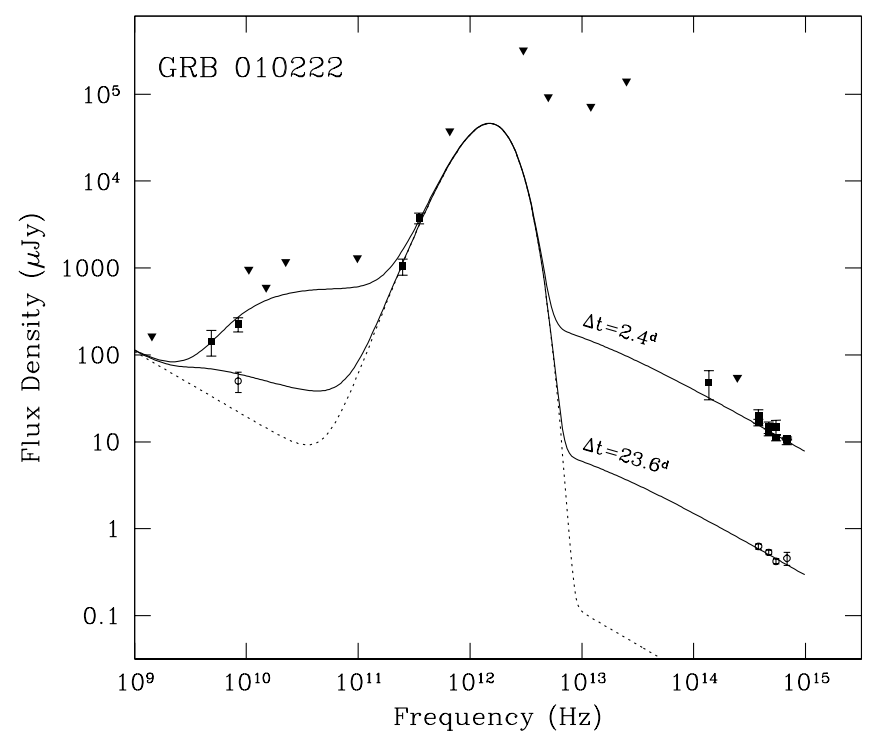

FIG. 2.- Spectral energy distribution (SED) toward GRB 010222. The dashed line is the estimated contribution of the ULIRG SMM $\mathrm{J} 14522+4301$ to the total emission. It consists of a modified blackbody of the form $F_{v} \propto B_{v}(T)\left\{1-\exp \left[-\left(v / v_{0}\right)^{\alpha}\right]\right\}$ (where $T=40 \mathrm{~K}, \alpha=1.5$, and $v_{0}=10^{15} \mathrm{~Hz}$ ) plus a power-law synchrotron spectrum of the form $F_{v} \propto v^{\alpha_{r}}$, where $\alpha_{r}=-0.75$ (see text for more details). To this starburst SED we have added the SED for the afterglow emission from GRB 010222 (solid lines) at two epochs, 2.4 and 23.6 days after the burst. These have been derived from broadband modeling of all available radio, optical, and X-ray measurements taken between 0.15 and 75 days after the burst ( $T$. J. Galama et al. 2001, in preparation). The millimeter and submillimeter flux density measurements are from Table 1 , the centimeter radio and NIR/ optical data are from Galama et al., and the IRAS data $(3 \sigma)$ were obtained from the NASA/IPAC InfraRed Science Archive. To better constrain the SED of SMM J14522 + 4301, we have used the average flux densities at $250 \mathrm{GHz}$ (IRAM) and $350 \mathrm{GHz}$ (JCMT) rather than single-epoch measurements. fails, however, because the source was readily detectable at centimeter wavelengths (Berger \& Frail 2001), where a simple extrapolation of this slope should suggest otherwise.

Such steep slopes are reminiscent of thermal emission from dust. At wavelengths longer than optical, the emissivity of dust grains is usually modeled as $Q(\lambda) \propto \lambda^{-\beta}$, where $1 \leq \beta \leq 2$. For dust that is optically thin in the RayleighJeans portion, this results is a spectrum of $F_{v} \propto v^{2+\beta}$, consistent with the observed steep slope. We begin by considering whether the dust could have been heated (and partially evaporated) by the burst itself or by the prompt optical/UV flash generated in the reverse shock of the afterglow (Waxman \& Draine 2000; Esin \& Blandford 2000; Reichart 2001; Venemans \& Blain 2001). The subsequent reprocessing of this radiation, either from dust scattering or by thermal emission, results in a long-lived infrared emission component (or dust echo). A simple energy calculation demonstrates the difficulties with this interpretation. For our adopted cosmology $\left(H_{0}=65 \mathrm{~km} \mathrm{~s}^{-1} \mathrm{Mpc}^{-1}, \Omega_{M}=\right.$ 0.3 , and $\Lambda_{0}=0.7$ ) the luminosity distance corresponding to the lower redshift limit of $z=1.477$ (Jha et al. 2001) is $d_{L}=$ $3.56 \times 10^{28} \mathrm{~cm}$. The isotropic luminosity at $350 \mathrm{GHz} \propto$ $v F_{v}$ (defined in the rest frame) is $L_{v} \simeq 5 \times 10^{44} \mathrm{ergs} \mathrm{s}^{-1}$. Within 8 days of observation, the total energy emitted directly into the $350 \mathrm{GHz}$ band is of order $10^{50}$ ergs. This dust emission is expected to be roughly isotropic, so there should be no geometric correction even if the burst itself was beamed. More importantly, the bolometric corrections are expected to be huge. Calculations by Waxman \& Draine (2000) and Venemans \& Blain (2001) show that dust is sublimated by the optical/UV flash at any distance closer than about $10 \mathrm{pc}$, and the surviving dust just outside this distance is heated to $2000 \mathrm{~K}$ with a spectral peak at a rest-frame wavelength of $1-10 \mu \mathrm{m}$. In this case the bolometric correction to $L_{v}$ at $350 \mathrm{GHz}$ is more than 6 orders of magnitude. Even in the most optimistic case, the optical/ UV flash is expected to be only a small fraction of the GRB energy (Sari \& Piran 1999; Waxman \& Draine 2000), and therefore it is not enough to account for the total energy radiated at $350 \mathrm{GHz}$ during the duration of our observations. If one hypothetically sets up a geometrical situation in which the dust is located farther away from the burst to allow for lower temperatures and therefore smaller bolometric correction to the $350 \mathrm{GHz}$ flux, one finds that the emission will last for years, increasing again the required energy. We therefore conclude the GRB is not energetic enough to create the submillimeter flux by heating its surrounding dust.

We are led to a model in which the millimeter and submillimeter emission is not dominated by the direct or reprocessed light from the afterglow itself, but rather originates as a distinct and separate component. The simplest hypothesis, consistent with the persistent nature of the source and its steep spectrum, is that we have detected a dusty, highredshift galaxy coincident with GRB 010222, designated as SMM J14522 + 4301. The population of submillimeter galaxies, of which SMM J14522 + 4301 is an example, produce the bulk of the submillimeter background and are an important contributor to star formation at high redshifts (Smail, Ivison, \& Blain 1997); such systems appear to be analogs of local ultraluminous infrared galaxies (ULIRGs; e.g., Sanders \& Mirabel 1996). In the next two sections we will examine this hypothesis in more detail and derive the physical properties of the galaxy. 


\section{SUBMILLIMETER AND MILLIMETER GALAXY PROPERTIES}

If GRB 010222 occurred in SMM J14522 + 4301, then its physical properties can be derived. The far-infrared (FIR) and submillimeter emission from SMM J14522+4301 originates from dust heated by an interstellar radiation field dominated by massive stars (Kennicutt 1998). In this instance we are well justified in representing the thermal dust emission spectrum by a modified blackbody that takes the form in the Rayleigh-Jeans regime of $F_{v} \propto v^{\alpha} B_{v}(T)$. A fit of the OVRO, IRAM, and JCMT flux-density measurements (and upper limits) results in a bolometric luminosity of $L_{\mathrm{Bol}}=4 \times 10^{12} L_{\odot}$. A dust temperature of $T_{d}=40 \mathrm{~K}$ and a dust emissivity index $\beta=1.5$ were assumed (Blain 1999). For a continuous starburst, the star formation rate $\mathrm{SFR} \simeq \delta_{\mathrm{MF}}\left(L_{\mathrm{Bol}} / 10^{10} L_{\odot}\right) M_{\odot} \mathrm{yr}^{-1}$ (Omont et al. 2001), where $\delta_{\mathrm{MF}}$ depends on the stellar mass function. At starburst ages of 10-100 Myr, $\delta_{\mathrm{MF}}$ lies between 0.8 and 2, so by adopting $\delta_{\mathrm{MF}}=1.5$ we derive $\mathrm{SFR} \simeq 600 \quad M_{\odot} \quad \mathrm{yr}^{-1}$. Although the uncertainties are at least a factor of 2 , these values of SFR and $L_{\text {Bol }}$ place SMM J14522+4301 in the class of ULIRGs (Smail et al. 1997).

With what degree of confidence can we assume that SMM J14522+4301 is the host galaxy of GRB 010222? From submillimeter source counts of dusty galaxies (Blain et al. 1999), background source confusion is expected at the flux level of SMM J14522+4301 in the 14".5 (FWHM) JCMT beam at $350 \mathrm{GHz} 2 \% \pm 1 \%$ of the time. Given the surfeit of NIR/optical candidates in Figure 3, it is not sufficient to secure the identification of GRB 010222 with SMM $\mathrm{J} 14522+4301$ on a posteriori grounds alone. Similar claims have been made for an Infrared Space Observatory (ISO) detection of a persistent source toward GRB 970508 at 60 $\mu \mathrm{m}$ (Hanlon et al. 2000). In our view, independent corroborative evidence is needed before any such associations can be made.

The problem here is a common one. Because of the large beams used in their detection, source confusion poses considerable difficulties for identifying the optical counterparts of submillimeter-selected galaxies (e.g., Smail et al. 2000a). In addition to a detection coincident with GRB 010222,

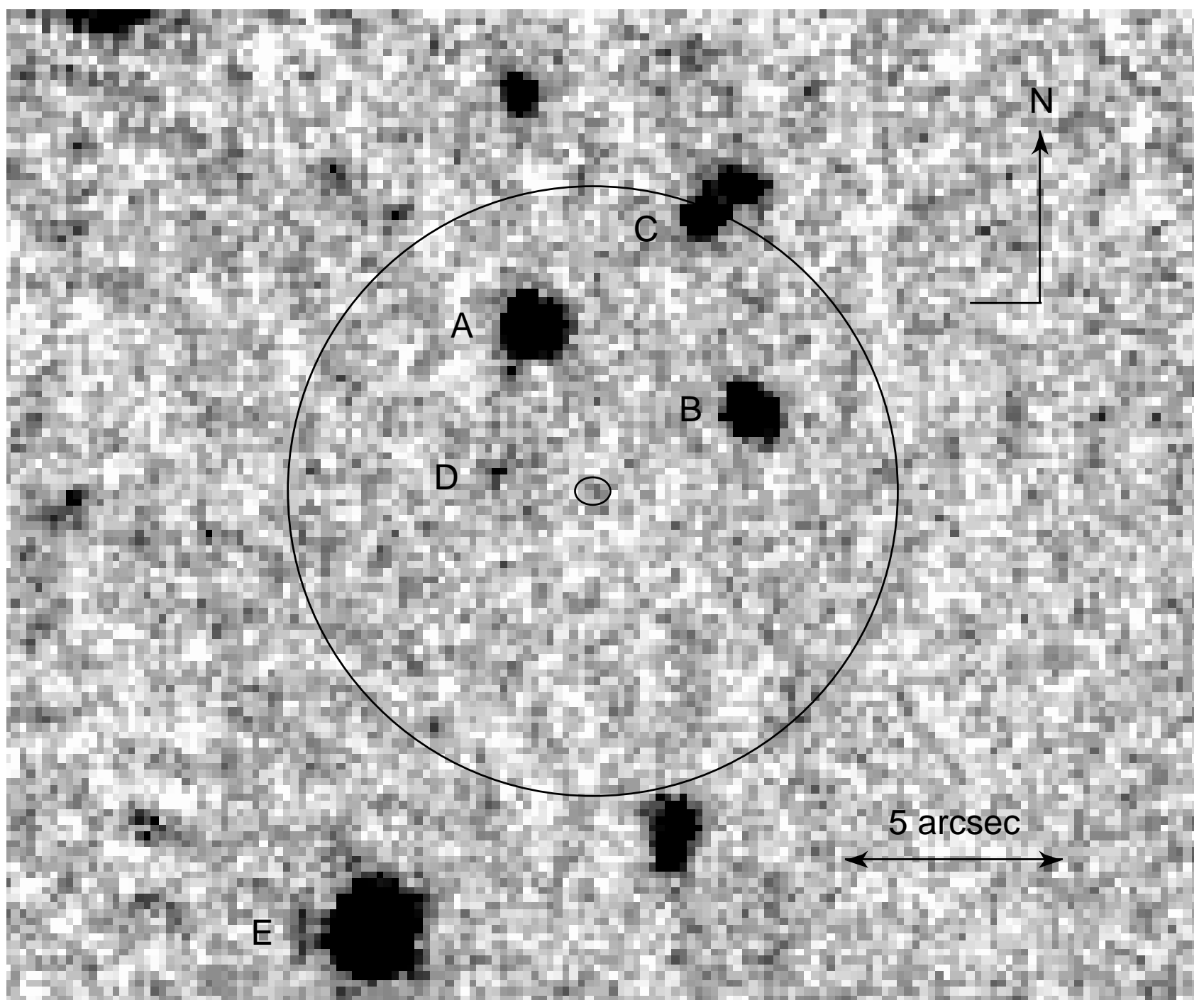

FIG. 3. - K-band Keck II + NIRPSEC image of the field of GRB 010222. The 7" radius of the SCUBA beam is indicated and the four galaxies (A-D) discussed in the text are labeled. Also marked is the $3 \sigma$ error ellipse of the optical transient based on a relative astrometric solution with the HST + WFPC2 third-epoch F814W observation of the afterglow. The host of GRB 010222 is detected at the $3.6 \sigma$ level. North is up, and east is to the left. 
there are four other objects in the $K$-band image that lie within the JCMT beam (Fig. 3 and Table 2). Deep observations $(I>26$ and $K>21)$ toward submillimeter sources have revealed a growing number of highly obscured sources (EROs) with very red colors $I-K \gtrsim 5$ (Smail et al. 1999; Dey et al. 1999; Frayer et al. 2000). Object "B," 4" away from GRB 010222, is the reddest galaxy in the error circle with $\mathrm{F} 814 \mathrm{~W}-K \simeq 4.5$, nominally qualifying it as an ERO. The red colors of ERO galaxies may be due to an old stellar population or a highly reddened population of young stars. Their space density, although not well determined, is comparable to that of SCUBA sources at these flux levels (Cowie et al. 1994; Moustakas et al. 1997; Smail et al. 1999).

Not all submillimeter galaxies are red objects, however. A case in point is SMM J14011+0252 at $z=2.56$ (Ivison et al. 2000), which consists of two components (J1 and J2), neither of which is especially red, $I-K \sim 3$. Still others, such as SMM J02399-0136 at $z=2.80$ (Ivison et al. 1998) and SMM J02399-0134 at $z=1.06$ (Soucail et al. 1999), have blue colors. This diversity of optical properties is also reflected in samples of local ULIRGs, which have spectral energy distributions (SEDs) that when shifted to high $z$ can be as blue as optically selected field galaxies (Trentham, Kormendy, \& Sanders 1999).

The detection of centimeter radiation coincident with submillimeter sources has proved to be a powerful method for identifying counterparts (Smail et al. 2000b). Likewise, the most secure identification of an ULIRG with a GRB is that of Berger et al. (2001), where a persistent centimeter source was found spatially coincident with GRB 980703 . The method relies on the well-known radio/FIR correlation for star-forming galaxies (Condon 1992). The thermal dust emission at $350 \mathrm{GHz}$ due to massive stars can be related to the synchrotron and thermal bremsstrahlung emission at $1.4 \mathrm{GHz}$ via a redshift-dependent spectral index $\alpha_{1.4}^{350}$ (Carilli \& Yun 1999). Using the relation of Carilli \& Yun (1999), we find a value of $\alpha_{1.4}^{350}=0.69 \pm 0.16$ at $z=1.477$ and predict a centimeter flux density at $1.4 \mathrm{GHz}$ of $8585_{-50}^{+115} \mu \mathrm{Jy}$. T. J. Galama et al. (2001, in preparation) have noted a flattening of the 8.46 and $4.86 \mathrm{GHz}$ light curves at late times. This deviation from model predictions of the afterglow flux is significant at the 2.5 and $2.3 \sigma$ levels, respectively. For a centimeter spectral index of $\alpha=-0.7$, typical of such galaxies, we find that within the errors the centimeter emission is consistent with an ULIRG at $z \simeq 1.5$. There are no other radio sources visible in the JCMT beam to an $\mathrm{rms}$ of $\sim 6 \mu \mathrm{Jy}$ beam ${ }^{-1}$ with a $\sim 1^{\prime \prime}$ beam at $8.46 \mathrm{GHz}$. Continued monitoring will verify whether this is the centimeter detection of the host galaxy of GRB 010222.

In the absence of suitable optical/NIR color discriminants, submillimeter sources have been identified by searching for molecular gas at the candidate redshift (e.g., Frayer et al. 1998) or by better constraining the position with a interferometric detection of the millimeter continuum (e.g., Bertoldi et al. 2000). If SMM J14522+4301 is an ULIRG at the same redshift of GRB 010222, then it is estimated from the observed flux density at $250 \mathrm{GHz}$ (and assuming $T_{d}=$ $40 \mathrm{~K}, \beta=1.5$, and dust-to-gas ratio equal to 100 ) that there is a total gas mass of $3 \times 10^{10} M_{\odot}$ (see Omont et al. 2001 and references therein) - an amount that is readily detectable with current instruments. A future search for redshifted CO (2-1) at $z=1.477$ would be a promising means to confirm the association.

\section{OPTICAL AND NIR GALAXY PROPERTIES}

The current evidence identifies the host galaxy of GRB 010222 as the most likely counterpart of SMM $\mathrm{J} 14522+4301$. The argument is supported on probabilistic grounds and, more importantly, the tentative detection of the host at centimeter wavelengths (§ 5). The optically visible portion of the host galaxy of GRB 010222 is blue, with $\mathrm{F} 814 \mathrm{~W}-K \simeq 2.1$. The rest-frame $B$-band luminosity of this galaxy $(z=1.477)$ is $M_{B}=-18.6$. We have used the formalism of Lilly et al. (1995) and assume $C(B, I)=-0.2$ for the color term of the $K$-correction, appropriate for latetype galaxies. This galaxy is similar to the host galaxy of GRB 970228 (Bloom, Djorgovski, \& Kulkarni 2001)—both are subluminous $\left(L \sim 0.1 L_{*}\right)$ relative to $L_{*}$ galaxies at their respective redshifts. Neither the magnitude nor the luminosity of this galaxy, however, is exceptional compared to other GRB host galaxies (Mao \& Mo 1998; Hogg \& Fruchter 1999; Schaefer 2000). The $M_{B}$ luminosity corresponds to a rest-frame blue luminosity $L_{B} \simeq v L_{v}=2 \times 10^{9} L_{\odot}$, which is small compared to the bolometric luminosity of $L_{\mathrm{Bol}}=4$ $\times 10^{12} L_{\odot}$ derived in $\S 5$. Despite the prodigious star formation, the distribution of dust in the host galaxy must consist of regions of significant internal extinction while still allowing the escape of some UV light.

\section{DISCUSSION AND CONCLUSIONS}

Multiwavelength studies of GRBs and their host galaxies have the potential both to determine the fraction of star formation that is optically obscured (Wijers et al. 1998; Totani 1999; Blain \& Natarajan 2000) and to elucidate the relation between the optical/UV and submillimeter-selected galaxies. To the degree that GRBs trace massive star formation in the early universe (Bloom et al. 1999; Galama et al. 2000; Piro et al. 2000; Bloom, Kulkarni, \& Djorgovski 2000; Reichart 2001), then the extreme luminosity and the dust-penetrating ability of $\gamma$-rays can be used to select highredshift galaxies in an unbiased way without regard to their emission properties. Surveys for optical/UV galaxies are magnitude-limited while, in contrast, the sample of GRB host galaxies have magnitudes $V \sim 22-28$ that span the complete range of model predictions (Hogg \& Fruchter 1999). Obtaining reliable galaxy identifications is an ongoing problem for submillimeter sources, but the early detection of an afterglow ensures that both an accurate position and (in many cases) a redshift will be known for the galaxy.

In this paper we have identified a persistent, steepspectrum submillimeter source and concluded that it is a dusty starburst galaxy, most likely the host galaxy of GRB 010222. Although centimeter and submillimeter observations of GRB host galaxies have only just begun (Smith et al. 1999; Berger et al. 2001; Hanlon et al. 2000), they are revealing aspects of the sample that were not apparent from more extensive optical/NIR observations. The detection of significant submillimeter and centimeter radiation suggests that at least some GRB host galaxies are undergoing prodigious star formation $\left(>500 M_{\odot} \mathrm{yr}^{-1}\right)$. Such results are important as they are a direct link between GRBs and episodes of massive star formation (Berger et al. 2001).

Future deep submillimeter and centimeter radio observations of GRB host galaxies hold some promise for exploring these differences in their optical/UV properties. The current sample is somewhat limited. Centimeter obser- 
vations have not been carried out to the requisite level (i.e., at $z=1,10 \mu \mathrm{Jy} \simeq 10 M_{\odot} \mathrm{yr}^{-1}$ at $1.4 \mathrm{GHz}$ ), and to do so for the entire sample would require the sensitivity of the Expanded VLA (EVLA), currently under development. Submillimeter and millimeter observations have been undertaken toward 10 well-localized GRBs (Shepherd et al. 1998; Bremer et al. 1998; Kulkarni et al. 1999; Smith et al. 1999; Frail et al. 2000a; Berger et al. 2000), but for the most part the existing upper limits would not be sufficient to rule out galaxies as bright as SMM J14522+4301. RamirezRuiz, Trentham, \& Blain (2002) have calculated that $20 \%$ of GRB host galaxies are detectable at $350 \mathrm{GHz}$ with the SCUBA array on JCMT, a number that is close to the current detection rate.

We thank all the observers on the JCMT whose programs were displaced in order to enable these target-of- opportunity observations. We are grateful to E. Kreysa and the MPIfR bolometer team for providing MAMBO and to R. Zylka for the MOPSI software package. Thanks also to the IRAM staff for allowing flexible scheduling at the $30 \mathrm{~m}$. This work was supported in part by grants from the NSF, NASA, and private foundations to S. R. K., S. G. D., F. A. H., and R. S.; Fairchild Fellowships to R. S. and T. J. G.; Hubble Fellowship to D. E. R.; Millikan Fellowship to A. D.; and a Hertz Fellowship to J. S. B. IRAM is supported by INSU/CNRS (France), MPG (Germany), and IGN (Spain). Research at the Owens Valley Radio Observatory is supported by the National Science Foundation through grant AST 96-13717. D. A. F. thanks Rob Ivison and C. Carilli for useful conversations and an anonymous JCMT referee for the catchy title. This research has made use of NASA's Astrophysics Data System Abstract Service.

\section{REFERENCES}

Berger, E., et al. 2000, ApJ, 545, 56

Berger, E., \& Frail, D. A. 2001, GCN Circ. 968 (http://gcn.gsfc.nasa.gov/ $\operatorname{gcn} / \operatorname{gcn} 3 / 968 . g c n 3)$

Berger, E., Kulkarni, S. R., \& Frail, D. A. 2001, ApJ, 560, 652

Bertoldi, F., et al. 2000, A\&A, 360, 92

Blain, A. W. 1999, MNRAS, 309, 955

Blain, A. W., Kneib, J.-P., Ivison, R. J., \& Smail, I. 1999, ApJ, 512, L87

Blain, A. W., \& Natarajan, P. 2000, MNRAS, 312, L35

Bloom, J. S., et al. 1999, Nature, 401, 453

Bloom, J. S., Djorgovski, S. G., \& Kulkarni, S. R. 2001, ApJ, 554, 678

Bloom, J. S., Kulkarni, S. R., \& Djorgovski, S. G. 2000, AJ, submitted (astro-ph/0010176)

Bremer, M., Krichbaum, T. P., Galama, T. J., Castro-Tirado, A. J., Frontera, F., Van Paradijs, J., Mirabel, I. F., \& Costa, E. 1998, A\&A, 332, L13

Carilli, C. L., \& Yun, M. S. 1999, ApJ, 513, L13

Cimatti, A., Andreani, P., Rottgering, H., \& Tilanus, R. 1998, Nature, 392, 895

Condon, J. J. 1992, ARA\&A, 30, 575

Cowie, L. L., Gardner, J. P., Hu, E. M., Songaila, A., Hodapp, K., \& Wainscoat, R. J. 1994, ApJ, 434, 114

Dey, A., Graham, J. R., Ivison, R. J., Smail, I., Wright, G. S., \& Liu, M. C. 1999, ApJ, 519, 610

Esin, A. A., \& Blandford, R. 2000, ApJ, 534, L151

Frail, D. A., et al. 2000a, ApJ, 538, L129

. 2000b, in AIP Conf. Proc. 526, 5th Huntsville Symp. on GammaRay Bursts, ed. R. M. Kippen, R. S. Mallozzi, \& G. J. Fishman (New York: AIP), 298

Frayer, D. T., Ivison, R. J., Scoville, N. Z., Yun, M., Evans, A. S., Smail, I., Blain, A. W., \& Kneib, J. 1998, ApJ, 506, L7

Frayer, D. T., Smail, I., Ivison, R. J., \& Scoville, N. Z. 2000, AJ, 120, 1668

Galama, T. J., et al. 2000, ApJ, 536, 185

Hanlon, L., et al. 2000, A\&A, 359, 941

Henden, A. 2001, GCN Circ. 961 (http://gcn.gsfc.nasa.gov/gcn/gcn3/ 961.gcn3)

Hogg, D. W., \& Fruchter, A. S. 1999, ApJ, 520, 54

Holland, W. S., et al. 1999, MNRAS, 303, 659

Ivison, R. J., Smail, I., Barger, A. J., Kneib, J., Blain, A. W., Owen, F. N., Kerr, T. H., \& Cowie, L. L. 2000, MNRAS, 315, 209

Ivison, R. J., Smail, I., Le Borgne, J., Blain, A. W., Kneib, J., Bezecourt, J., Kerr, T. H., \& Davies, J. K. 1998, MNRAS, 298, 583

Jenness, T., \& Lightfoot, J. F. 1998, in ASP Conf. Ser. 145, Astronomical

Data Analysis Software \& Systems VII, ed. R. Albrecht, R. N. Hook, \&

H. A. Bushouse (San Francisco: ASP), 216

Jenness, T., Lightfoot, J. F., \& Holland, W. S. 1998, Proc. SPIE, 3357, 548

Jha, S., et al. 2001, ApJ, 554, L155
Katz, J. L., \& Piran, T. 1997, ApJ, 490, 772

Kennicutt, R. C. 1998, ARA\&A, 36, 189

Kreysa, E., et al. 1998, Proc. SPIE, 3357, 319

Kulkarni, S. R., et al. 1999, ApJ, 522, L97

Lilly, S. J., Tresse, L., Hammer, F., Crampton, D., \& Le Fevre, O. 1995, ApJ, 455, 108

Mao, S., \& Mo, H. J. 1998, A\&A, 339, L1

McDowell, J. 2001, GCN Circ. 963 (http://gcn.gsfc.nasa.gov/gcn/gcn3/ 963.gcn3)

Moustakas, L. A., Davis, M., Graham, J. R., Silk, J., Peterson, B. A., \& Yoshii, Y. 1997, ApJ, 475, 445

Omont, A., et al. 2001, A\&A, in press

Persson, S. E., Murphy, D. C., Krzeminski, W., Roth, M., \& Rieke, M. J. 1998, AJ, 116, 2475

Piro, L. 2001a, GCN Circ. 960 (http://gcn.gsfc.nasa.gov/gcn/gcn3/ 960.gcn 3 )

961. 2001b, GCN Circ. 959 (http://gcn.gsfc.nasa.gov/gcn/gcn3/ 961.gcn3)

Piro, L., et al. 2000, Science, 290, 955

Ramirez-Ruiz, E., Trentham, N., \& Blain, A. W. 2002, MNRAS, 329, 465

Reichart, D. E. 2001, ApJ, 554, 643

Sanders, D. B., \& Mirabel, I. F. 1996, ARA\&A, 34, 749

Sari, R., \& Piran, T. 1999, ApJ, 520, 641

Schaefer, B. E. 2000, ApJ, 533, L21

Shepherd, D. S., Frail, D. A., Kulkarni, S. R., \& Metzger, M. R. 1998, ApJ, 497,859

Smail, I., Ivison, R. J., \& Blain, A. W. 1997, ApJ, 490, L5

Smail, I., Ivison, R., Blain, A., \& Kneib, J.-P. 2000a, in Proc. UMass/ INAOE Conf., Deep Millimeter Surveys, ed. J. Lowenthal \& D. Hughes (Singapore: World Scientific), in press

Smail, I., Ivison, R. J., Kneib, J., Cowie, L. L., Blain, A. W., Barger, A. J., Owen, F. N., \& Morrison, G. 1999, MNRAS, 308, 1061

Smail, I., Ivison, R. J., Owen, F. N., Blain, A. W., \& Kneib, J. 2000b, ApJ, 528,612

Smith, I. A., et al. 1999, A\&A, 347, 92

Soucail, G., Kneib, J. P., Bézecourt, J., Metcalfe, L., Altieri, B., \& Le Borgne, J. F. 1999, A\&A, 343, L70

Totani, T. 1999, ApJ, 511, 41

Trentham, N., Kormendy, J., \& Sanders, D. B. 1999, AJ, 117, 2152

Venemans, B. P., \& Blain, A. W. 2001, MNRAS, 325, 1477

Waxman, E. 1997, ApJ, 489, L33

Waxman, E., \& Draine, B. T. 2000, ApJ, 537, 796

Wijers, R. A. M. J., Bloom, J. S., Bagla, J., \& Natarajan, P. 1998, MNRAS, 294, L13

Zylka, R. 1998, MOPSI Users Manual (Grenoble: IRAM) 